University of New Orleans

ScholarWorks@UNO

$9-1988$

\title{
Division-of-wave-front thin-film beam splitter for generating binary patterns of orthogonal elliptical polarization states
}

\author{
R. M.A. Azzam \\ University of New Orleans, razzam@uno.edu
}

Follow this and additional works at: https://scholarworks.uno.edu/ee_facpubs

Part of the Electrical and Electronics Commons, and the Optics Commons

\section{Recommended Citation}

R. M. A. Azzam, "Division-of-wave-front thin-film beam splitter for generating binary patterns of orthogonal elliptical polarization states," J. Opt. Soc. Am. A 5, 1576-1580 (1988)

This Article is brought to you for free and open access by the Department of Electrical Engineering at ScholarWorks@UNO. It has been accepted for inclusion in Electrical Engineering Faculty Publications by an authorized administrator of ScholarWorks@UNO. For more information, please contact scholarworks@uno.edu. 


\title{
Division-of-wave-front thin-film beam splitter for generating binary patterns of orthogonal elliptical polarization states
}

\author{
R. M. A. Azzam \\ Department of Electrical Engineering, University of New Orleans, Lakefront, New Orleans, Louisiana 70148
}

Received February 1, 1988; accepted March 22, 1988

\begin{abstract}
A division-of-wave-front thin-film beam splitter is described that reflects monochromatic light at oblique incidence with orthogonal elliptical polarization states. It consists of a metallic substrate partially covered with a transparent thin film that inverts the ratio $\rho$ of the complex $p$ and $s$ reflection coefficients at the principal angle of the metal. Any pattern of coated and uncoated areas of the substrate is imprinted upon the reflected wave front as a corresponding two-dimensional spatial binary polarization pattern. A specific design is given that uses a Au substrate at a wavelength of $632.8 \mathrm{~nm}$. The effects of small errors in the film refractive index, the film thickness, and the angle of incidence are discussed. It is noted that a layer that inverts $\rho$ at a certain (especially high) angle of incidence is an effective $\rho$-inverting layer at all angles.
\end{abstract}

\section{INTRODUCTION}

Recently ${ }^{1,2}$ I described a new optical device that consists of a dielectric or absorbing (semiconductor or metallic) substrate coated by a transparent thin film of a certain refractive index and of a thickness that alternates between two specific values over adjacent areas of the substrate. When a monochromatic plane wave of light is incident upon the device at a certain angle (such that the wave is refracted in the film at $45^{\circ}$ ), the two-dimensional (2-D) binary thickness pattern (obtained, e.g., by lithographic techniques) is imprinted upon the reflected wave front as a 2-D pattern of orthogonal linear ( $p$ and $s$ ) polarization states. Subsequently ${ }^{3}$ this concept was extended to the generation of 2-D spatial binary patterns of orthogonal, right- and left-handed, circular polarizations by using an optically dense transparent substrate (e.g., Ge in the IR) on which $+90^{\circ}$ and $-90^{\circ}$ thin-film reflection quarter-wave retarders are integrated (i.e., reside side by side) and the incident light is linearly polarized at a $45^{\circ}$ azimuth from the plane of incidence.

In this paper such a reflective division-of-wave-front thinfilm beam splitter (DOW TF BS) is generalized further to produce orthogonal elliptical polarization states.

\section{DIVISION-OF-WAVE-FRONT THIN-FILM BEAM SPLITTER FOR ORTHOGONAL ELLIPTICAL POLARIZATION STATES}

Operation of the DOW TF BS is explained with reference to Fig. 1. A monochromatic plane wave of light is incident from a transparent ambient of refractive index $N_{0}$ (usually air, for which $N_{0}=1$ ) onto the planar surface of an absorbing substrate of complex refractive $N_{2}$, which is covered partially by a transparent thin film of refractive index $N_{1}$ and uniform thickness $d$. All media are assumed to be linear, homogeneous, isotropic, and nonmagnetic. The angle of incidence is chosen to be the principal angle $\phi$ of the substrate. In terms of the complex relative dielectric constant $\epsilon$ $=\left(N_{2} / N_{0}\right)^{2}=\left(\epsilon_{r}, \epsilon_{i}\right), \phi$ is obtained by solving the cubic equation ${ }^{4}$

$$
a_{3} u^{3}+a_{2} u^{2}+a_{1} u+a_{0}=0,
$$

where

$$
\begin{aligned}
& a_{0}=\epsilon_{r}^{2}+\epsilon_{i}^{2}, \\
& a_{1}=-2\left(\epsilon_{r}^{2}+\epsilon_{i}^{2}\right)-2 \epsilon_{r}, \\
& a_{2}=\left(\epsilon_{r}^{2}+\epsilon_{i}^{2}\right)+4 \epsilon_{r}+1, \\
& a_{3}=-2 \epsilon_{r}-2,
\end{aligned}
$$

and $u=(\sin \phi)^{2}$.

Let $\rho=r_{p} / r_{s}$ be the ratio of complex $p$ and $s$ reflection coefficients. At $\phi$, the $\rho$ value of the uncoated substrate becomes pure imaginary,

$$
\rho=\rho_{u}=j \tan \psi,
$$

where $\psi$ is called the principal azimuth.

The refractive index and the thickness $\left(N_{1}\right.$ and $\left.d\right)$ of the film are chosen to invert $\rho^{5,6}$ so that, for the coated substrate,

$$
\rho=\rho_{c}=-j \cot \psi \text {. }
$$

It is convenient to represent the state of polarization of light by the complex number ${ }^{7}$

$$
\chi=E_{p} / E_{s}
$$

which is the ratio of the phasor components of the electric vector that are parallel $\left(E_{p}\right)$ and perpendicular $\left(E_{s}\right)$ to the plane of incidence. It follows immediately that, for the optically isotropic system under consideration, the reflected and incident polarizations are related by

$$
\chi_{r}=\rho \chi_{i} \text {. }
$$

We assume that the incident light is linearly polarized at a $45^{\circ}$ azimuth from the plane of incidence, so that $\chi_{i}=1$. From Eqs. (3), (4), and (6), the polarization states reflected by the uncoated and coated parts of the substrate are given by

$$
\chi_{r u}=j \tan \psi, \quad \chi_{r c}=-j \cot \psi
$$




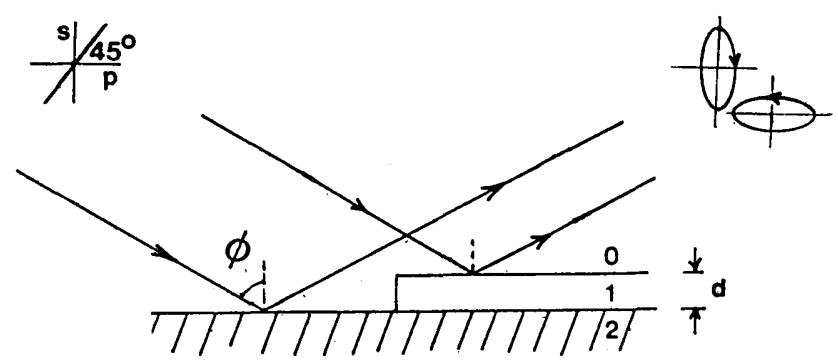

Fig. 1. DOW TF BS for generating orthogonal elliptical polarization states. Linearly polarized light at a $45^{\circ}$ azimuth from the plane of incidence is incident upon a metal substrate at the principal angle. The metal is partially coated by a transparent $\rho$-inverting layer.

Two polarization states $\chi_{1}$ and $\chi_{2}$ are orthogonal if they satisfy the condition ${ }^{7}$

$$
\chi_{1} \chi_{2}^{*}=-1 \text {, }
$$

where the asterisk denotes the complex conjugate. The two reflected polarizations given by Eqs. (7) do satisfy the orthogonality condition of Eq. (8). $\chi_{r u}$ and $\chi_{r c}$ represent rightand left-handed elliptical polarization states of the same axial ratio whose major axes are aligned perpendicular and parallel to the plane of incidence, respectively.

It is also of interest to calculate the intensity reflectances of the uncoated and coated areas of the substrate for the incident linearly polarized light at a $45^{\circ}$ azimuth, given by

$$
R_{k}=\left(r_{p k} r_{p k}^{*}+r_{s k} r_{s k}^{*}\right) / 2,
$$

where the subscripts $k=u$ and $c$ refer to the uncoated and coated areas, respectively.

In summary, a DOW TF BS can be designed to produce orthogonal elliptical polarizations by illuminating an absorbing substrate that is partially covered by a $\rho$-inverting layer with linearly polarized light that is incident at the principal angle and that has a vibration direction $45^{\circ}$ from the plane of incidence.

\section{SPECIFIC EXAMPLE}

As a concrete example of a DOW TF BS for the generation of orthogonal elliptical polarization states, we take a Au substrate and assume incident light of wavelength $\lambda=632.8 \mathrm{~nm}$ from a He-Ne laser. The complex refractive index of $\mathrm{Au}$ at this wavelength ${ }^{8}$ is $0.20-j 3.71$. Solving Eq. (1) gives the principal angle $\phi=75.8266^{\circ}$. At $\phi$, the reflectances of the Au substrate for $p$-polarized, $s$-polarized, and $45^{\circ}$ linearly polarized light are $0.8925,0.9873$, and 0.9399 , respectively, and the principal azimuth $\psi=\arctan \left(R_{p} / R_{s}\right)^{1 / 2}=43.5553^{\circ}$.

By a procedure already described in Reffs. 5 and 6, the refractive index and the thickness of the transparent $\rho$ - inverting layer at $\phi$ are calculated to be $N_{1}=1.9475$ and $d=$ $81.85 \mathrm{~nm}$. A suitable thin-film coating material is silicon nitride, the stoichiometry of which can be controlled ${ }^{9}$ to achieve this desired value of $N_{1}$. It should be noted that $d$ is the least film thickness; thicker $\rho$-inverting layers are obtained by adding any integral multiple of the film thickness period $D_{\phi}=187.33 \mathrm{~nm}$. The reflectances of the Au coated with the $\rho$-inverting layer for $p$-polarized, $s$-polarized, and $45^{\circ}$ linearly polarized light are $0.9399,0.8497$, and 0.8948 , respectively. The associated principal azimuth is $46.4447^{\circ}$, which is equal to $90^{\circ}-\psi$, as anticipated. Also, it can be verified by direct calculation that the differential reflection phase shift, $\Delta=\arg \rho$, equals $-90.0000^{\circ}$ and $+90.0000^{\circ}$, respectively, for the uncoated and coated parts of the substrate, as required by design.

The reflectances, for incident linearly polarized light at a $45^{\circ}$ azimuth, of the uncoated and coated areas of the substrate are sufficiently high (93.99 and 89.48\%, respectively) to make this device practical. Their difference of $4.5 \%$ produces a weak spatial-intensity modulation of the reflected wave front, which is superimposed upon, and registered with, the much more significant binary polarization modulation between orthogonal elliptical states.

For convenience, the important device characteristics are summarized in Table 1. For comparison, Table 1 also lists data obtained at another wavelength, $\lambda=488.0 \mathrm{~nm}$, of the $\mathrm{Ar}^{+}$-ion laser. Because of its diminished reflectance, $\mathrm{Au}$ is not a particularly good substrate at this shorter wavelength. Also, the reflectance difference between the coated and uncoated areas is relatively large and would cause appreciable intensity modulation of the reflected wave front.

\section{ERROR ANALYSIS}

In this section we examine the effects of small errors in the film refractive index $N_{1}$, the film thickness $d$, and the angle of incidence $\phi$ on the $\rho$-inversion condition $\left(\rho_{c} \rho_{u}=1\right)$, which is key to the operation of the DOW TF BS. The deviation of each of these parameters from its design value at $\lambda=632.8$ $\mathrm{nm}$ (listed in Table 1) is considered individually. Each parameter error causes a magnitude error (ME) and a phase error (PE) that are defined by

$$
\mathrm{ME}=\left|\rho_{c} \rho_{u}\right|-1, \quad \mathrm{PE}=\arg \left(\rho_{c} \rho_{u}\right),
$$

where $\rho_{c}$ is a function of $\left(N_{1}, d, \phi\right)$ and $\rho_{u}$ is a function of $\phi$.

Figure 2 shows the ME and PE caused by shifting $N_{1}$ by \pm 0.05 around the design value of 1.9475 . To keep $\mathrm{ME}<1 \%$ and $\mathrm{PE}<3^{\circ}$, the deviation $\Delta N_{1}$ should not exceed \pm 0.01 .

Figure 3 shows the $\mathrm{ME}$ and $\mathrm{PE}$ caused by thickness changes of $\pm 5 \mathrm{~nm}$ around the design value of $81.85 \mathrm{~nm}$. For $\mathrm{ME}<1 \%$ and $\mathrm{PE}<3^{\circ}, d$ should be controlled to within \pm 1

Table 1. Characteristics of DOW TF BS's Using a Au Substrate at Two Laser Wavelengths ${ }^{a}$

\begin{tabular}{ccccccccc}
\hline$\lambda(\mathrm{nm})$ & $n_{2}$ & $k_{2}$ & $\phi\left(^{\circ}\right)$ & $N_{1}$ & $d(\mathrm{~nm})$ & $\psi\left(^{\circ}\right)$ & $R_{u}$ \\
\hline 632.8 & 0.20 & 3.71 & 75.8266 & 1.9475 & 81.85 & 43.5553 & 0.9399 & 0.8948 \\
448.0 & 0.94 & 1.70 & 66.1446 & 2.5394 & 27.96 & 32.9133 & 0.5198 & 0.2070 \\
\hline
\end{tabular}

a $\lambda$ is the wavelo fractive index and the thickness of the $\rho$-inverting layer, $\psi$ is the substrate principal azimuth, and $R_{u}$ and $R_{c}$ are the reflectances for incident linearly polarized light at $a 45^{\circ}$ azimuth of the uncoated and coated parts of the Au substrate. 

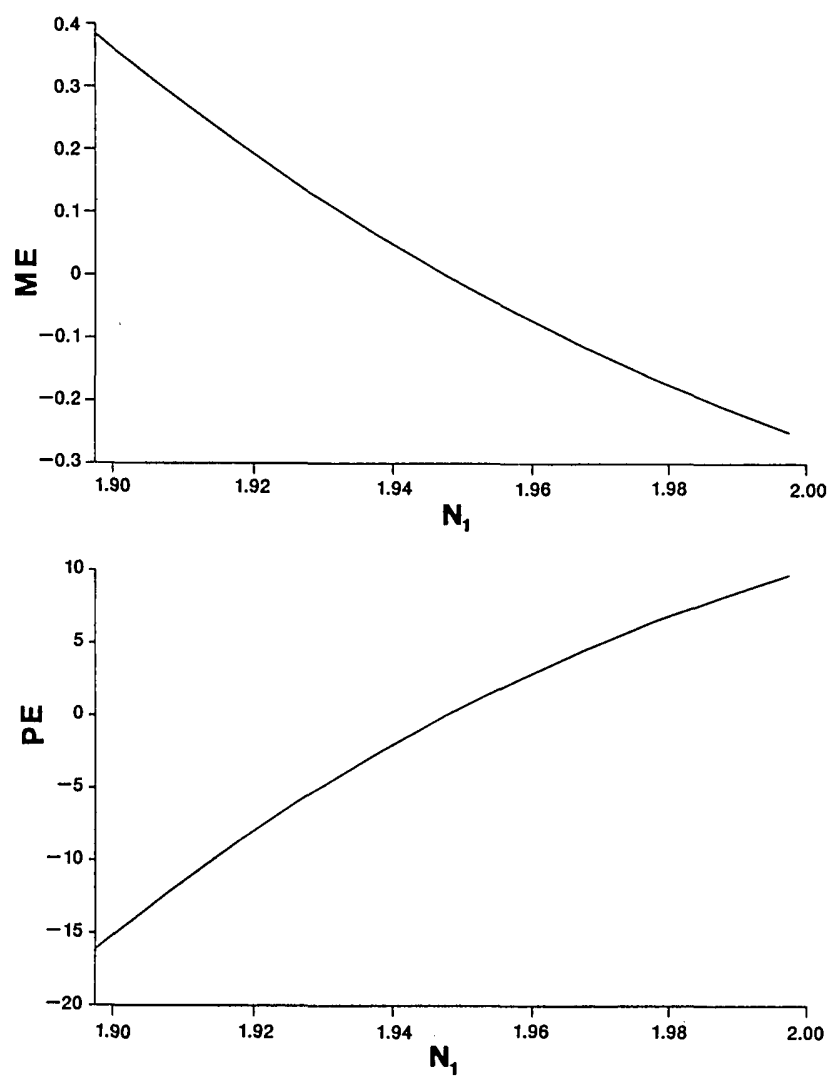

Fig. 2. $M E$ and $P E$ caused by shifting the refractive index of a transparent film on Au from the value (1.9475) required for $\rho$ inversion at the principal angle $\left(75.8266^{\circ}\right)$ and at wavelength of $632.8 \mathrm{~nm}$.
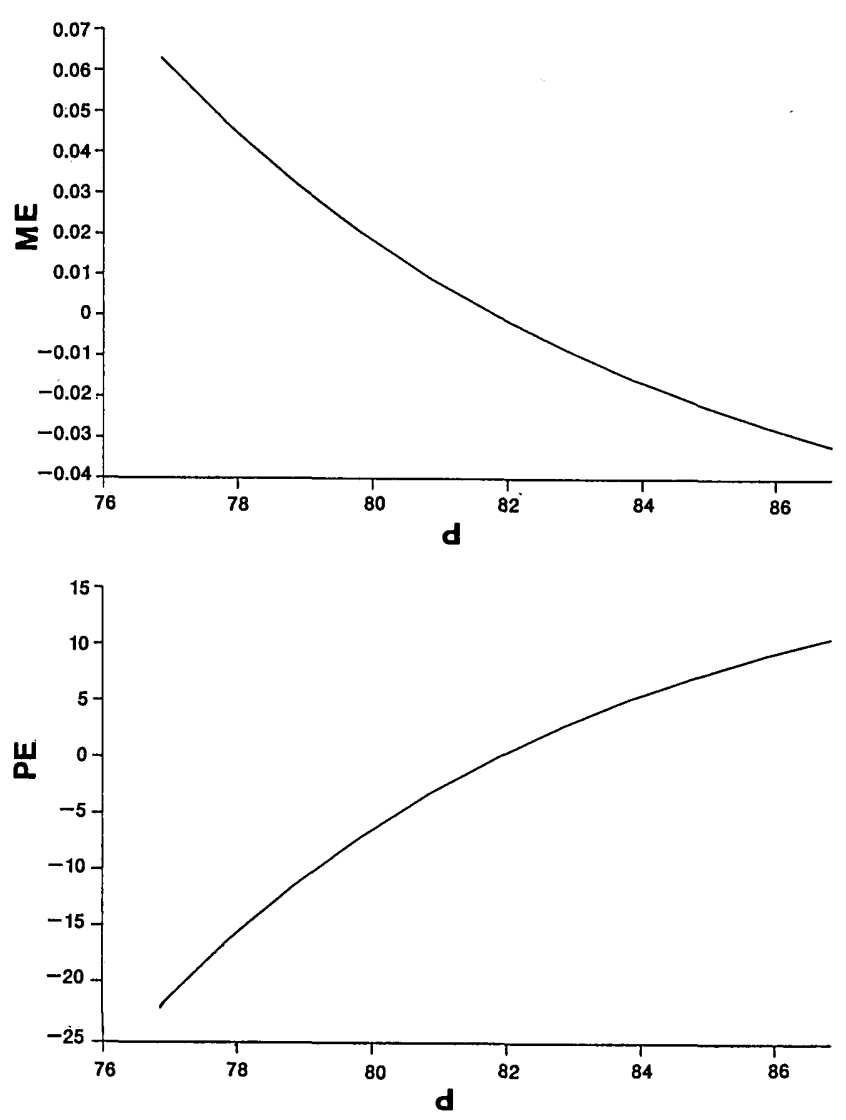

Fig. 3. Same as in Fig. 2, except that here the film thickness is shifted around the design value of $81.85 \mathrm{~nm}$.
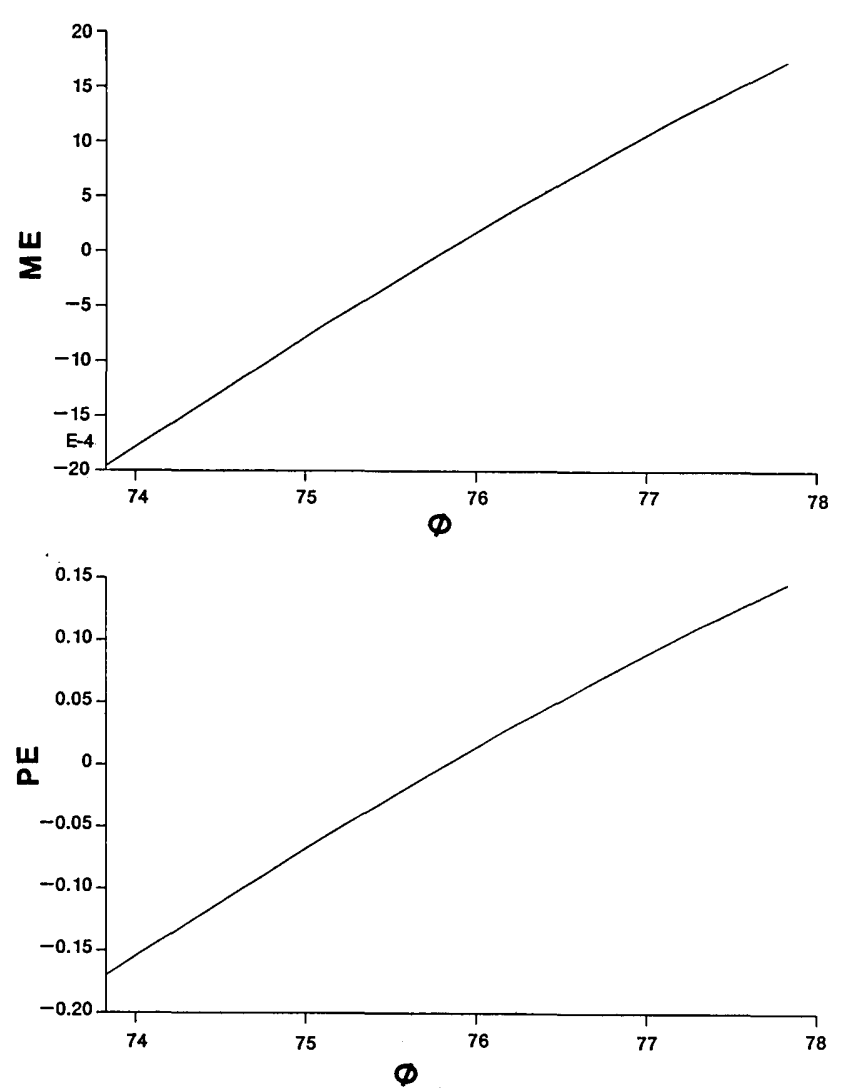

Fig. 4. Same as in Fig. 2, except that here the angle of incidence is shifted around the principal angle of $75.8266^{\circ}$.

nm. These tolerances on the refractive index and the thickness of the film are achievable with modern film-deposition technologies in conjunction with advanced ellipsometric monitoring.

Finally, Fig. 4 shows the $\mathrm{ME}$ and $\mathrm{PE}$ that result from shifting the angle of incidence by $\pm 2^{\circ}$ around the principal angle $\phi=75.8266^{\circ}$. It is remarkable that $\mathrm{ME}<2 \times 10^{-3}$ and $\mathrm{PE}<0.2^{\circ}$, so that appreciable angular errors do not substantially affect the $\rho$-inversion condition.

In Appendix A it is verified that $\rho$ inversion is maintained over nearly the entire range of $\phi$ from normal to grazing incidence. ${ }^{10}$

\section{CONCLUSION}

The DOW TF BS is a new optical device that embosses a binary pattern of orthogonal polarization states onto an optical wave front. This pattern corresponds to a binary pattern of thickness of a transparent thin film coating upon a transparent or absorbing substrate. In this paper previous work $^{1-3}$ that dealt with the generation of orthogonal linear and circular states is extended to the generation of orthogonal elliptical states. This is accomplished by reflecting light from a metallic (or semiconductor) substrate at the principal angle and using a $\rho$-inverting layer for the coating. A specific device based on a Au substrate is described for the common 632.8-nm He-Ne laser radiation, and the effects of small errors in the film refractive index, the film thickness, and the angle of incidence are considered.

The DOW TF BS can serve as a useful half-shade device. ${ }^{11}$ More importantly, it provides a new means of spatial binary 
polarization modulation for a new class of 2-D optical information processors that operate on polarization instead of the scalar complex field amplitude or intensity. It should also be noted that, if a spatially homogeneous analyzer is placed in the reflected wave front, a controllable binary intensity pattern can be obtained. For example, if the analyzer is crossed with one of the orthogonal reflected states, the binary levels of transmitted intensities will be zero (or a minimum) and a maximum.

For a review of other single-layer-coated optical devices for polarized light, the reader may consult Ref. 12. Other useful reviews that discuss applications of single-layer and multilayer thin films are given in Refs. 11 and 13.

\section{APPENDIX A}

In Section 4 and in Fig: 4 it is shown that $\rho$ inversion is affected negligibly by an angular swing of $\pm 2^{\circ}$ around $\phi$. This suggests that a layer that inverts $\rho$ at one angle of incidence may act as an effective $\rho$-inverting layer over a broad range of incidence angles. Figure 5 confirms this. It shows $\mathrm{ME}$ and $\mathrm{PE}$ versus $\phi$ over the entire range $0 \leq \phi \leq 90^{\circ}$ for the same transparent film on a Au substrate that inverts $\rho$ at the principal angle, with $\lambda=632.8 \mathrm{~nm}$. The maximum $\mathrm{ME}$ and $\mathrm{PE}$ values are $1.6 \%$ and $2^{\circ}$, respectively.

To determine whether this is perhaps a peculiarity of $\rho$ inversion at the principal angle only, we repeat the calculations for layers that invert $\rho$ at angles of incidence from 20 to $80^{\circ}$ in steps of $10^{\circ} . N_{1}$ and $d$ values of these layers are listed in Table 2. Again, we assume a Au substrate $\left(N_{2}=0.20-\right.$ j3.71) at $\lambda=632.8 \mathrm{~nm}$. Figures 6 and 7 show the $\mathrm{ME}$ and $\mathrm{PE}$
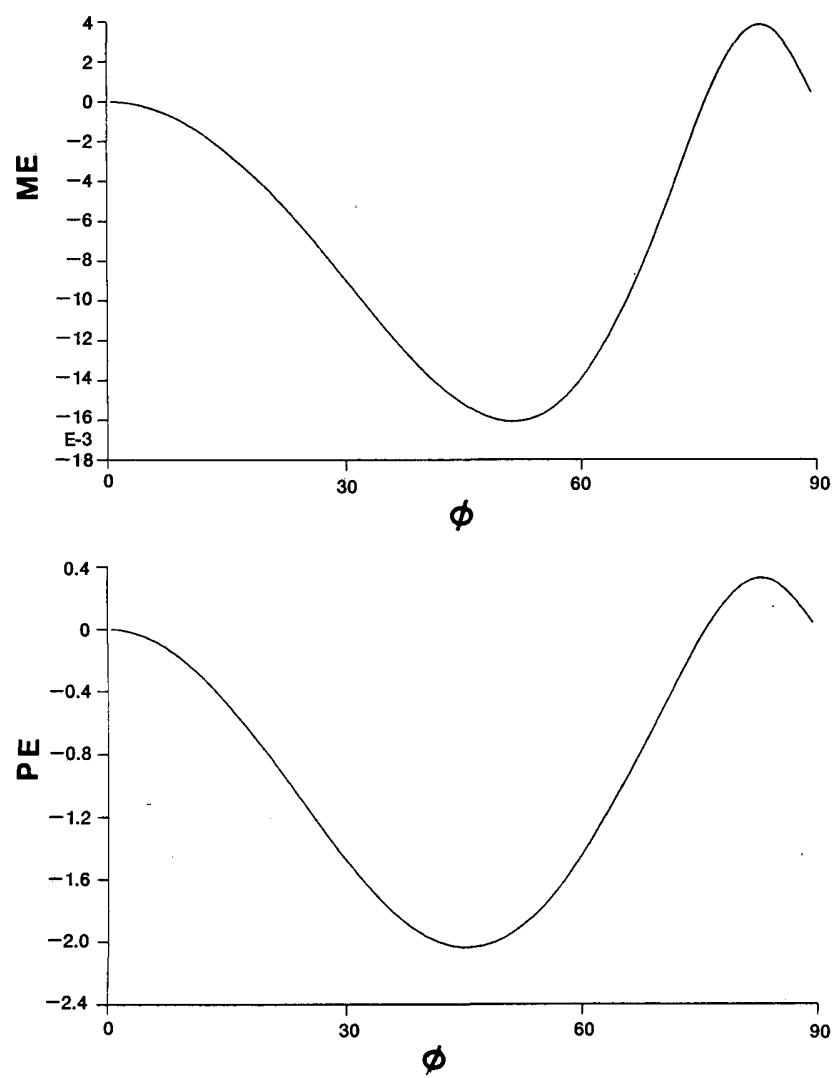

Fig. 5. Same as in Fig. 4, except that the entire range of incidence angles from 0 to $90^{\circ}$ is covered.
Table 2. Refractive Index $N_{1}$ and Thickness $d$ of a $\rho$ Inverting Layer on Au at Several Angles of Incidence $\phi^{a}$

\begin{tabular}{ccc}
\hline$\phi\left(^{\circ}\right)$ & $N_{1}$ & $d(\mathrm{~nm})$ \\
\hline 20 & 3.0195 & 38.26 \\
30 & 2.8295 & 42.60 \\
40 & 2.6116 & 48.69 \\
50 & 2.3925 & 56.50 \\
60 & 2.1914 & 65.88 \\
70 & 2.0226 & 76.22 \\
80 & 1.9064 & 85.27 \\
\hline
\end{tabular}

${ }^{a}$ We assume a wavelength of $632.8 \mathrm{~nm}$ and a corresponding complex refractive index of Au of $0.20-j 3.71$ (from Ref. 8).

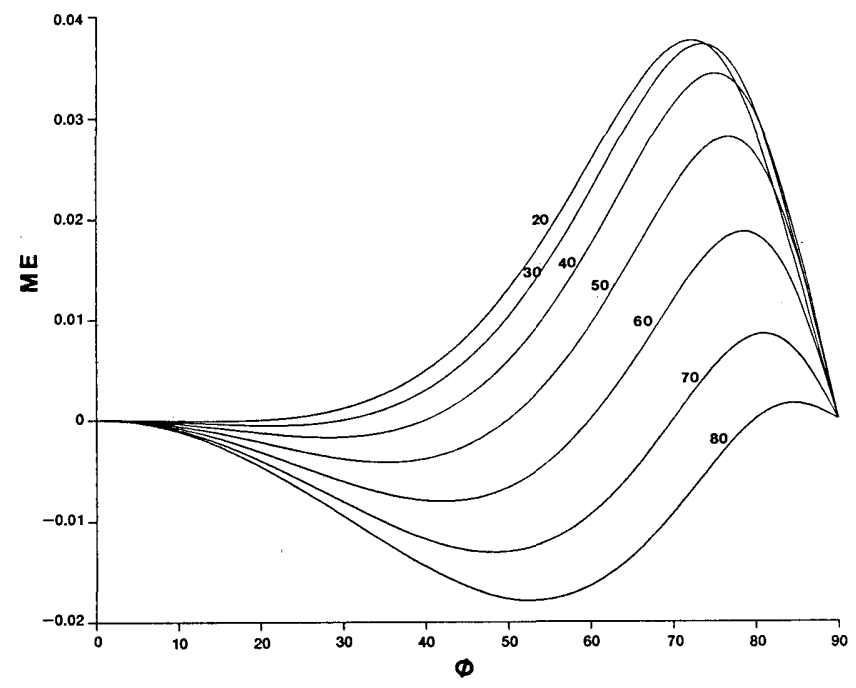

Fig. 6. ME as a function of the angle of incidence $\phi$ for different $\rho$ inverting layers on $\mathrm{Au}$ at a wavelength of $632.8 \mathrm{~nm}$. Each layer, whose refractive index and thickness are given in Table 2, inverts $\rho$ exactly at the angle of incidence marked by each curve.

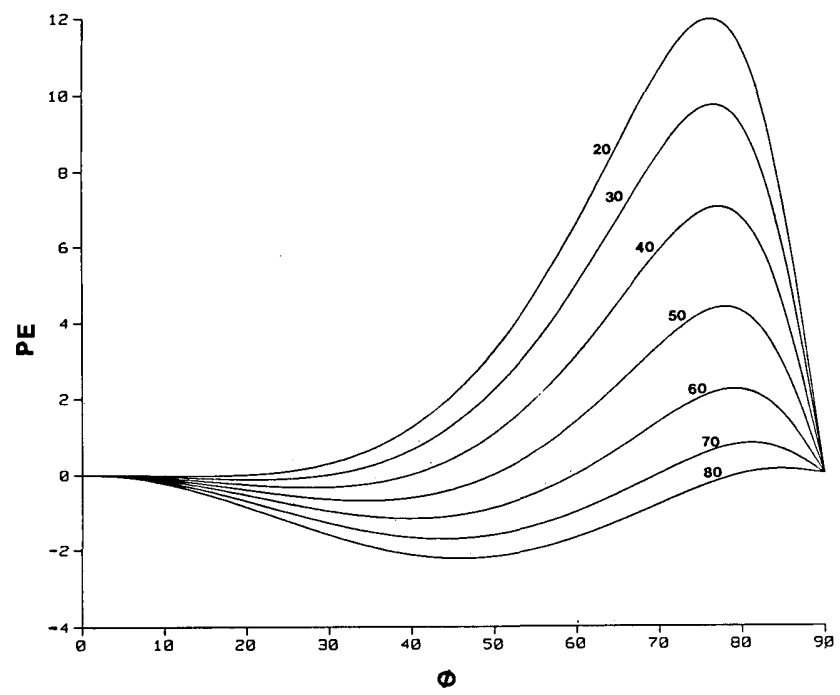

Fig. 7. Same as in Fig. 6, except that here PE is plotted versus $\phi$. 
values, respectively. It is apparent that the $\rho$-inversion condition is maintained reasonably well, to a good approximation, over the entire range of $\phi$. This is so especially for layers that invert $\rho$ at high angles, e.g., at $60^{\circ}, 70^{\circ}$, and $80^{\circ}$.

The reason for this broad angular insensitivity is that $\rho$ inversion is invariably satisfied at $\phi=0$ and $90^{\circ}$ as well as at the design angle of oblique incidence. This establishes three nodes of zero error in the ME-versus- $\phi$ and PE-versus$\phi$ curves, and the excursion of these errors becomes constrained between nodes.

\section{ACKNOWLEDGMENTS}

I am pleased to thank I. Elminyawi and M. Abu-Tahnat for their assistance with the calculations. This research was partially supported by the National Science Foundation under grant ECS8520035.

\section{REFERENCES AND NOTES}

1. R. M. A. Azzam, "Division-of-wavefront polarizing beam splitter and half-shade device using dielectric thin film on dielectric substrate," Appl. Opt. 23, 1296-1298 (1984).

2. R. M. A. Azzam, "Extinction of the $p$ and $s$ polarizations of a wave on reflection at the same angle from a transparent film on an absorbing substrate: applications to parallel-mirror crossed polarizers and a novel integrated polarimeter," J. Opt. Soc. Am. A 2, 189-197 (1985)
3. R. M. A. Azzam, "Reflective thin film device for generating spatial binary patterns of orthogonal circular polarizations," Opt. Commun. 55, 297-299 (1985).

4. R. M. A. Azzam, "Contours of constant principal angle and constant principal azimuth in the complex $\epsilon$ plane,"J. Opt. Soc. Am. 71, 1523-1528 (1981).

5. R. M. A. Azzam, "Inverting the ratio of the complex parallel and perpendicular reflection coefficients of an absorbing substrate using a transparent thin-film coating," J. Opt. Soc. Am. A 1, 699-702 (1984)

6. R. M. A. Azzam and M. A. Habli, "Constraint on the optical constants of a transparent film on an absorbing substrate for inversion of the ratio of complex $p$ and $s$ reflection coefficients at a given angle of incidence," Appl. Opt. 26, 4717-4721 (1987)

7. R. M. A. Azzam and N. M. Bashara, Ellipsometry and Polarized Light (North-Holland, Amsterdam, 1987), Sec. 1.7.

8. P. Joensen, J. C. Irwin, J. F. Cochran, and A. E. Curzon, "Transmission method for determining the optical constants of metals," J. Opt. Soc. Am. 63, 1556-1562 (1973).

9. G. Eisenstein and L. W. Stulz, "High quality antireflection coatings on laser facets by sputtered silicon nitride," Appl. Opt. 23, 161-164 (1984).

10. This, however, does not extend to polarization orthogonality, which holds at, and in the immediate vicinity of, the principal angle.

11. J. M. Bennett and H. E. Bennett, "Polarization," in Handbook of Optics, W. G. Driscoll and W. Vaughan, eds. (McGraw-Hill, New York, 1978), Sec. 10.

12. R. M. A. Azzam, "Single-layer-coated optical devices for polarized light," Thin Solid Films (to be published).

13. J. A. Dobrowolski, "Coatings and filters," in Handbook of Optics, W. G. Driscoll and W. Vaughan, eds. (McGraw-Hill, New York, 1978), Sec. 8. 